\title{
TEORES DE SILÍCIO NO SOLO E NA PLANTA DE ARROZ DE TERRAS ALTAS COM DIFERENTES DOSES DE ADUBAÇÃO SILICATADA E NITROGE NADA(1)
}

\author{
M. MAUAD(2), H. GRASSI FILHO(3), \\ C. A. C. CRUSCIOL ${ }^{(4)} \&$ J . C. CORRÊA ${ }^{(2)}$
}

\begin{abstract}
RESUMO
O Si não é elemento essencial para o crescimento e desenvolvimento das plantas, porém sua absorção pode trazer inúmeros benefícios para culturas acumuladoras de $\mathrm{Si}$, como o arroz. Entretanto, considerando o avançado grau de intemperização em que se encontram os solos tropicais, os teores de $\mathrm{Si}$ disponível nestes solos são baixos. $\mathbf{O}$ objetivo deste trabal ho foi avaliar, na cultura do arroz de terras altas sob condições de túnel plástico, o efeito de doses de Si e de $\mathbf{N}$ na produção de matéria seca, na produtividade de grãos, no teor de $\mathrm{N}$, nos teores de Si no solo e na planta e na quantidade de Si extraído do solo. Os tratamentos foram constituídos por três doses de $\mathrm{N}\left(5,75\right.$ e $\left.150 \mathrm{mg} \mathrm{kg}^{-1} \mathrm{de} N\right)$, tendo como fonte a uréia e quatro doses de Si $\left(0,200,400\right.$ e $\left.600 \mathrm{mg} \mathrm{kg}^{-1} \mathrm{de} \mathrm{SiO}_{2}\right)$ tendo como fonte o silicato de cálcio (Wollastonita). 0 delineamento experimental utilizado foi inteiramente casualizado com esquema fatorial $3 \times 4$, com cinco repetições. $\mathbf{O}$ acúmulo de matéria seca, a produtividade de grãos e os teores de $\mathbf{N}$ na planta não foram influenciados pelas doses de Si. $\mathbf{O}$ incremento da adubação nitrogenada aumentou a produção de matéria seca, a produtividade de grãos e o teor de $\mathbf{N}$ na planta, porém nenhum efeito foi encontrado para os teores de Si no solo. Houve interação N x Si para os teores de Si na planta e para a quantidade de $\mathrm{Si}$ acumulado pelas plantas.
\end{abstract}

Termos de indexação: Oryza Sativa L., silício, nitrogênio.

(1) Parte da Tese de Mestrado em Agricultura, apresentada, pelo primeiro autor à Faculdade de Ciências Agronômicas da Universidade Estadual Paulista - UNESP. Projeto financiado pela FAPESP no 99/08300-4. Recebido para publicação em março de 2002 e aprovado em agosto de 2003.

(2) Engenheiro-Agrônomo, Departamento de Produção Vegetal-Setor de Agricultura, Faculdade de Ciências Agronômicas da U niversidade Estadual Paulista - UNESP. Caixa Postal 237, CEP 18609-690 Botucatu (SP). E-mail mauad@aser.com.br

(3) Professor Adjunto, Departamento de Recursos Naturais-Setor de Ciência do Solo, Faculdade de Ciências Agronômicas, UNESP. Bolsista CNPq. E-mail: heliograssi @fca.unesp.br

(4) Professor Adjunto, Departamento de Produção Vegetal-Setor de Agricultura, Faculdade de Ciências Agronômicas, UNESP. Bolsista CNPq. E-mail: crusciol @fca.unesp.br 


\title{
SUMMARY: SILICON CONTENTS IN SOIL AND IN HIGHLAND RICE PLANTS UNDER DIFFERENT DOSES OF SILICON AND NITROGEN FERTILIZATION
}

\begin{abstract}
Theel ement Silicon is not deemed essential for plant growth and devel opment, but its absorption can benefit cumulativecul tures likericegreatly. TheSi content of tropical soils, however, is very low, dueto theadvanced weathering degree. Theobjectiveof this work was to eval uate the growth of rice plants in highlands under plastic tunnels and the effects of nitrogen $(\mathrm{N})$ and Si doses on dry matter production, grain productivity, $\mathrm{N}$ content; Si soil and plant contents; and on the amount of extracted silicon. The treatments consisted of three doses of $\mathrm{N}\left(5,75\right.$, and $150 \mathrm{mg} \mathrm{kg}^{-1}$ of soil $)$ in urea form and four doses of $\mathrm{SiO}_{2}(0,200$, 400 , and $600 \mathrm{mg} \mathrm{kg}^{-1}$ of soil) in calcium silicate form (Wollastonita). The completely randomized experimental design in a $3 \times 4$ factorial schemewas carried out in fivereplications. Dry matter accumulation, grain yiel ds, and $\mathrm{N}$ contents in the plant were not influenced by the silicon doses. Increased nitrogen fertilization raised the dry matter production, productivity grains, and $\mathrm{N}$ plant contents, but had no effect on Si soil contents. Therewas an $\mathrm{N} \times \mathrm{Si}$ interaction in relation to the Si plant content and theamount of $\mathrm{Si}$ accumulated by the plant.
\end{abstract}

Index terms: Oryza Sativa L., nitrogen, silicon.

\section{INTRODUÇÃO}

$\mathrm{O} \mathrm{Si}$, depois do $\mathrm{O}_{2}$, é o el emento mais abundante da crosta terrestre. Mesmo não sendo considerado elemento essencial para o desenvolvimento das plantas, sua absorção pode trazer inúmeros benefícios, principalmente para culturas acumuladoras deSi, como o arroz (Mengel \& Kirkby, 1987).

As plantas absorvem o Si da solução do solo na forma de ácido monossilícico $\mathrm{Si}(\mathrm{OH})_{4}$ (Tisdale et al., 1993). O óxido de silício $\left(\mathrm{SiO}_{2}\right)$ é o mineral mais abundante nos solos, constituindo a base da estrutura da mai oria dos argilominerais; entretanto, em razão do avançado grau de intemperização em que seencontram os sol os tropicais, oSi éencontrado basicamentena forma de quartzo, opala $\left(\mathrm{SiO}_{2} \cdot \mathrm{nH}_{2} \mathrm{O}\right)$ e outras formas não-disponíveis às plantas (Barbosa Filho et al., 2001).

A queda de produtividade do arroz em várias regiões do mundo está relacionada com vários fatores, dentre eles a baixa disponibilidade de nutrientes ou de elementos benéficos, como oSi. No caso do $\mathrm{Si}$, alguns fatores podem estar envolvidos na baixa disponibilidade nos solos para as plantas, tais como: (a) muitos solos de áreas produtoras de arroz de regi ões tropicais esubtropicais apresentam graus variados de dessilificação; (b) a cinética de dissolução do Si no solo é muito baixa, e (c) o Si da solução do solo é adsorvido por sesquióxidos que estão presentes em muitos solos tropicais (Savant et al., 1997).

O uso do Si tem promovido melhora na arquitetura da planta e aumento na fotossíntese
(Deren et al., 1994), resultado da menor abertura do ângulo foliar, que torna as folhas mais eretas, diminuindo o auto-sombreamento, sobretudo em condições de altas densidades populacionais e altas doses de N (Yoshida et al., 1962; Balastra et al., 1989). Além disso, promove o aumento da resistência da planta à incidência de doenças fúngicas, por ser tal elemento depositado na fol ha, nos tecidos da epiderme logo abaixo da cutícula, precisamente nas paredes cel ulares mais externas (Agarie et al., 1998), conferindo-Ihe resistência mecânica à penetração das hifas (Barbosa Filho et al., 2001).

Quanto à produção de matéria seca da parte aérea, tem-se verificado que a aplicação de Si não altera essa variável na cultura do arroz (Tanaka \& Park, 1966; Liang et al., 1994; Carvalho, 2000); porém, quanto à produtividade de grãos, a aplicação do elemento tem proporcionado resultados divergentes, ou seja, há relatos deincremento(Deren et al., 1994; Liang, 1994; Barbosa Filho et al., 1998; Korndörfer et al., 1999a; Faria, 2000) e de ausência de resposta (Carvalho, 2000).

Contudo, a ausência de resposta à aplicação de Si verificada por alguns autores, em solos considerados com teores baixos do elemento, pode estar relacionada, dentre várias causas, com a carência de informações de cultivares quanto à exigência eà capacidade de extração. Essa hipótese é ressaltada por Wislow (1992) e Barbosa Filho et al. (1998), que relataram que há diferença genotípica quanto à capacidade de absorver Si. Assim, a el evação nos teores do el emento no solo para níveis considerados adequados passa a ser importante, uma vez que não se conhece a exigência dos cultivares de arroz em relação ao $\mathrm{Si}$, tampouco o fato de os 
rizicultores utilizarem em suas áreas, em média, três materiais genéticos, sendo, pelo menos um, trocado a cada duas a três safras.

Elemento essencial para o crescimento e desenvol vimento das plantas, oN, quando fornecido em altas doses, pode diminuir os teores de Si nas plantas de arroz. Em experimento com arroz, aveia e tomate, Wallace (1989) observou que o incremento da adubação nitrogenada provocou redução nos teores de Si nas plantas de arroz e aveia.

O objetivo deste trabalho foi estudar, em condições de túnel plástico, os efeitos da adubação nitrogenada e silicatada na produção de matéria seca, na produtividade de grãos, no teor de $\mathrm{N}$ e nos teores de Si no solo e na planta de arroz cultivada em terras altas.

\section{MATERIAL E MÉTODOS}

O experimento foi instalado em túnel plástico, no período de 30/11/99 a 25/03/00, em área experimental pertencente ao Departamento de Produção Vegetal da Faculdade de Ciências Agronômicas - UNESP - Campus de Botucatu. O solo utilizado no experimento foi classificado como Latossolo Vermel ho distrófico (EMBRAPA, 1999), e a análise química, realizada segundo métodos propostos por Raij \& Quaggio (1983), apresentou os seguintes resultados: $\mathrm{P}=1 \mathrm{mg} \mathrm{dm}^{-3} ; \mathrm{M} . \mathrm{O}=16 \mathrm{~g} \mathrm{dm}^{-3}$, $\mathrm{pH}=4,0 ; \mathrm{H}+\mathrm{Al}=64,4 \mathrm{mmol}_{\mathrm{c}} \mathrm{dm}^{-3} ; \mathrm{K}^{+}=$ $0,14 \mathrm{mmol}_{\mathrm{C}} \mathrm{dm}^{-3} ; \mathrm{Ca}^{2+}=2,3 \mathrm{mmol}_{\mathrm{C}} \mathrm{dm}^{-3} ; \mathrm{Mg}^{2+}=$ $0,5 \mathrm{mmol}_{\mathrm{C}} \mathrm{dm}^{-3}, \mathrm{Al}^{+3}=4 \mathrm{mmol}_{\mathrm{C}} \mathrm{dm}^{-3} \mathrm{eV}=4,36 \%$. A calagem foi realizada 60 dias antes da semeadura de modo a elevar o valor $V$ para $50 \%$ (Raij et al., 1996), utilizando-se cal cário dolomítico com PRNT de $85 \%$.

Adotou-se o delineamento experimental inteiramente casualizado, com esquema fatorial $3 \times 4$, com cinco repetições, totalizando 60 unidades experimentais. Cada unidade experimental foi constituída por vaso com dimensões internas de $40 \times 40 \times 25 \mathrm{~cm}$, com $30 \mathrm{~kg}$ de solo e 2 linhas de semeadura de $0,40 \mathrm{~m}$ de comprimento e espaçadas de 0,20 m entre si. O cultivar de arroz utilizado foi IAC-202.

Os tratamentos constituíram da aplicação de 5, 75 e $150 \mathrm{mg} \mathrm{kg}^{-1}$ de N no solo, utilizando uréia como fonte de $\mathrm{N}$, e $0,200,400$ e $600 \mathrm{mg} \mathrm{kg}^{-1} \mathrm{de} \mathrm{SiO}_{2}$ no solo, o equivalente a $0,93,187$ e $280 \mathrm{mg} \mathrm{kg}^{-1} \mathrm{de} \mathrm{Si}$. Utilizou-se, como fonte de Si, silicato de cálcio (Wollastonita), com as seguintes características químicas e físicas: total de óxido de silício $\left(\mathrm{SiO}_{2}\right)=$ $452 \mathrm{~g} \mathrm{~kg}^{-1}$; óxido de silício $\left(\mathrm{SiO}_{2}\right)$ solúvel em ácido cítrico a 2 dag $\mathrm{L}^{-1}=318 \mathrm{~g} \mathrm{~kg}^{-1} ; \mathrm{pH}$ em água $=7,0 \mathrm{a}$ 7,5; solubilidade em água, desprezível; silicato de cálcio $=910 \mathrm{~g} \mathrm{~kg}^{-1}$; óxidos de Al, Fe, Mg eK $=10 \mathrm{~g} \mathrm{~kg}^{-1}$ e sais de flúor $=28,5 \mathrm{~g} \mathrm{~kg}^{-1}$.
A semeadura foi realizada no dia 30/11/99, utilizando 70 sementes viáveis por linha de semeadura. Aos seis dias da semeadura, quando $50 \%$ das plântulas de cada unidade experimental apresentavam o coleópti lo acima do nível do solo, considerou-sea data de emergência. Realizou-se um desbaste nove dias após a emergência, de modo a manter 30 plantas por linha de semeadura.

$\mathrm{Na}$ adubação de semeadura, foram aplicados: $5 \mathrm{mg} \mathrm{kg}^{-1}$ de $\mathrm{N}$ em todos os vasos, sendo utilizados a uréia ( $450 \mathrm{~g} \mathrm{~kg}^{-1}$ de N); $100 \mathrm{mg} \mathrm{kg}^{-1}$ de P e $150 \mathrm{mg} \mathrm{kg}^{-1}$ de $\mathrm{K}$, utilizando-se superfosfato simples (180 $\mathrm{g} \mathrm{kg}^{-1}$ de $\left.\mathrm{P}_{2} \mathrm{O}_{5}\right)$ e cloreto de potássio $\left(600 \mathrm{~g} \mathrm{~kg}^{-1}\right.$ de $\left.\mathrm{K}_{2} \mathrm{O}\right)$. Aos 35 dias da emergência, foi realizada adubação de cobertura com nitrogênio, utilizando 70 e $145 \mathrm{mg} \mathrm{kg}^{-1}$ de $\mathrm{N}$, de forma que o experimento ficasse com três diferentes doses de $\mathrm{N}$, sendo $\mathrm{N}_{1}=5 \mathrm{mg} \mathrm{kg}^{-1}$ de $\mathrm{N}, \mathrm{N}_{2}=75 \mathrm{mg} \mathrm{kg}^{-1}$ de $\mathrm{N}$ e $\mathrm{N}_{3}=150 \mathrm{mg} \mathrm{kg}^{-1}$ de $\mathrm{N}$. O Si foi incorporado ao solo 30 dias antes da semeadura, seguindo recomendação de Korndörfer et al. (1999a).

Foram coletadas 50 fol has bandeiras ao acaso de cada unidade experimental no período do florescimento, quando $50 \%$ das panículas estavam visíveis (Raij et al., 1996). O material col etado foi seco em estufa a $60^{\circ} \mathrm{C}$, até atingir peso constante, e posteriormente moído. Na matéria seca das fol has, foi determinado o teor de N, segundo Malavolta et al. (1997). O teor de Si no solo e na planta (colmo + fol ha) foi determinado no final do experimento, segundo método proposto por Korndörfer et al. (1999b). A quantidade de Si acumulado na planta foi calculada, utilizando os dados de teor de Si na planta e quantidade de matéria seca produzida por metro quadrado. Foi usada uma das linhas de semeadura para determinar a produção de matéria seca da parte aérea e produtividade de grãos. Os resultados obtidos, em cada variável analisada, foram submetidos à análise de variância, eas médias dos tratamentos foram comparadas pelo Teste de Tukey a $5 \%$.

\section{RESULTADOS E DISCUSSÃO}

A produção de matéria seca da parte aérea foi significativamenteaumentada com oincremento das doses da adubação nitrogenada aplicadas ao solo (Quadro 1). O N participa de diversos processos na planta, dentreel es a divisão celular ea constituição detecidos (Malavol ta et al., 1997). Assim, o aumento na produção de matéria seca obti do pel o incremento da adubação nitrogenada é devido à participação deste nutriente na produção de tecido vegetal.

As doses aplicadas de Si não proporcionaram alterações significativas na produção de matéria seca. Esses resultados estão deacordo com os relatos de Tanaka \& Park (1966), Liang et al. (1994) e 
Carvalho (2000), que também não encontraram diferença estatística.

Analisando os dados de produtividade de grãos apresentados no quadro 1 , observa-se que a maior produtividade ocorreu com a dose de $75 \mathrm{mg} \mathrm{kg}^{-1}$ de $\mathrm{N}$ eque o aumento, acima dessa dose, não foi seguido por um incremento na produtividade, mas, sim, por uma redução. Altas doses de $\mathrm{N}$ estimularam o perfil hamento ea formação denovas fol has, causando sombreamento, condições favoráveis a doenças, acamamento e queda na produtividade (Malavolta \& Fornasieri Filho, 1983; Barbosa Filho, 1987).

Nas condições experimentais, não ocorreram acamamento e doenças; assim, a queda na produtividade com o aumento da adubação nitrogenada, provavel mente, pode ser decorrente da soma de diversos fatores, em especial de um el evado número de colmos, que, apesar de não ter sido determinado, visualmente foi notado, que propiciou uma condição de sombreamento, diminuindo, assim, a área fotossinteticamente ativa. Com isso a planta não teve car boidratos suficientes para o enchimento de todos as espiguetas, tornando a produtividade menor. A maior produtividadealcançada com a dose de $75 \mathrm{mg} \mathrm{kg}^{-1}$ de $\mathrm{N}$ foi atribuída ao melhor equilíbrio no desenvolvimento da planta, que não acarretou sombreamento, tendo assim fotoassimilados suficientes para o enchimento das espiguetas.

As doses de Si não influíram na produtividade de grãos do arroz. Estes resultados discordam dos encontrados por Faria (2000), Korndörfer et al. (1999a), Deren et al. (1994) e Liang et al. (1994); entretanto, estão de acordo com os de Carvalho (2000), que, trabalhando com o mesmo cultivar utilizado neste estudo (IAC-202), em condições de campo, também não obteve aumentos significativos.

Cultivares como o IAC-202, do grupo moderno, tendem a responder mais à adubação nitrogenada do que os cultivares do grupo tradicional, os quais, por sua vez, apresentam mai or eficiência na absorção de Si (Winslow, 1992). Portanto, cabe lembrar que os genótipos de arroz diferem bastante quantoà sua capacidade de absorver Si (Winslow, 1992; Barbosa Filho et al., 1998). Apesar de não-significativo, observou-se um aumento da ordem de $8 \%$ ou $479 \mathrm{~kg} \mathrm{ha}^{-1}$ na produtividade de grãos, quando foram utilizados $400 \mathrm{mg} \mathrm{kg}^{-1}$ deSiO $_{2}$ em relaçãoà dosezero (Quadro 1).

Os teores de N na fol ha bandeira (Quadro 1) para todas as doses da adubação nitrogenada estiveram sempre na faixa apresentada por Raij et al. (1996) como sendo adequada (27-35 $\mathrm{g} \mathrm{kg}^{-1}$ ), porém aumentaram com a el evação da dose aplicada. Esse resultado foi decorrente do aumento da disponibilidade de $\mathrm{N}$ e, apesar deter proporcionado incremento na produção de matéria seca, também foi suficiente para el evar a concentração do el emento no tecido vegetal.

Quanto às doses de $\mathrm{Si}$, essas não afetaram os teores de N, ficando os valores do elemento dentro da faixa adequada, o que permite inferir que as quantidades de Si aplicadas não foram elevadas o suficiente para reduzir os teores de $\mathrm{N}$ na planta.

As doses deN nãoinfluenciaramsignificativamente os teores de ácido monossilícico [Si $\left.(\mathrm{OH})_{4}\right]$ no solo (Quadro 1). O Si $(\mathrm{OH})_{4}$ éum ácido fraco, de pequena força iônica (McKeague \& Cline, 1963; Raij \&

Quadro 1. Produção de matéria seca, produtividade de grãos, teor de nitrogênio na folha bandeira e teores de silício no solo, na planta e quantidade de silício acumulado nas plantas de acordo com a aplicação de $\mathbf{N}$ e Si

\begin{tabular}{|c|c|c|c|c|c|c|}
\hline Tratamento & $\begin{array}{l}\text { Matéria } \\
\text { seca }\end{array}$ & $\begin{array}{l}\text { Produtividade } \\
\text { de grãos }\end{array}$ & $\mathbf{N}$ & $\begin{array}{l}\text { Silício no } \\
\text { solo }\end{array}$ & $\begin{array}{l}\text { Silício na } \\
\text { planta }\end{array}$ & $\begin{array}{c}\text { Silício } \\
\text { acumulado }\end{array}$ \\
\hline Doses de $\mathrm{N}\left(\mathrm{mg} \mathrm{kg}^{-1}\right)$ & \multicolumn{2}{|c|}{$\mathrm{kg} \mathrm{ha}^{-1}$} & $\mathrm{~g} \mathrm{~kg}^{-1}$ & $\mathrm{mg} \mathrm{dm}^{-3}$ & $\mathrm{~g} \mathrm{~kg}^{-1}$ & $\mathrm{~kg} \mathrm{ha}^{-1}$ \\
\hline $\begin{array}{r}5 \\
75 \\
150\end{array}$ & $\begin{array}{l}5.371 \mathrm{c} \\
7.371 \mathrm{~b} \\
8.491 \mathrm{a}\end{array}$ & $\begin{array}{l}5.114 \mathrm{c} \\
7.890 \mathrm{a} \\
6.440 \mathrm{~b}\end{array}$ & $\begin{array}{l}27,6 \mathrm{c} \\
30,0 \mathrm{~b} \\
33,5 \mathrm{a}\end{array}$ & $\begin{array}{l}20,0 \\
21,9 \\
24,5\end{array}$ & $\begin{array}{l}26,2 \\
13,2 \\
16,6\end{array}$ & $\begin{array}{r}13,82 \\
9,93 \\
9,86\end{array}$ \\
\hline $\begin{array}{l}\text { Doses de } \mathrm{SiO}_{2} \text { (mg kg } \\
0 \\
200 \\
400 \\
600\end{array}$ & $\begin{array}{l}6.981 \\
6.780 \\
7.490 \\
6.990\end{array}$ & $\begin{array}{l}6.180 \\
6.500 \\
6.650 \\
6.500\end{array}$ & $\begin{array}{l}30,6 \\
30,3 \\
30,6 \\
30,0\end{array}$ & $\begin{array}{l}5,9 \mathrm{c} \\
14,0 \mathrm{bc} \\
23,5 \mathrm{~b} \\
45,1 \mathrm{a}\end{array}$ & $\begin{array}{r}8,5 \\
15,5 \\
19,4 \\
24,7\end{array}$ & $\begin{array}{r}55,8 \\
93,4 \\
137,3 \\
157,8\end{array}$ \\
\hline $\begin{array}{l}\text { Valores de F } \\
\text { Doses de N } \\
\text { Doses de Si } \\
\mathrm{N} \times \mathrm{Si}\end{array}$ & $\begin{array}{c}57,75^{* *} \\
1,53^{\mathrm{ns}} \\
1,43^{\mathrm{ns}}\end{array}$ & $\begin{array}{c}16,48^{* *} \\
0,28^{\text {ns }} \\
0,88^{\text {ns }}\end{array}$ & $\begin{array}{c}42,21^{* *} \\
0,29^{\mathrm{ns}} \\
1,66^{\mathrm{ns}}\end{array}$ & $\begin{array}{c}0,99^{\text {ns }} \\
40,77^{* *} \\
0,95^{\text {ns }}\end{array}$ & $\begin{array}{r}131,3^{* *} \\
72,2^{* *} \\
6,9^{* *}\end{array}$ & $\begin{array}{c}32,11 * * \\
90,19 * * \\
4,08 * *\end{array}$ \\
\hline C.V. (\%) & 1,33 & 23,6 & 6,7 & 46,3 & 18,3 & 16,73 \\
\hline
\end{tabular}

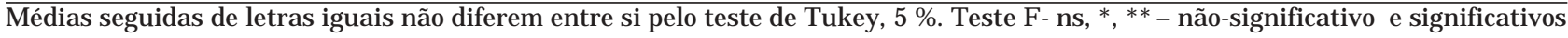
a 5 e $1 \%$. 
Camargo, 1973) e que, estando em solução, não compete com o nitrato por sítios de ligações no solo.

O menor teor deSi no solo 5,9 mg dm $^{3}$ (Quadro 1) foi encontrado na dose zero de $\mathrm{SiO}_{2}$, referindo-se, portanto, ao Si existente no solo. O baixo teor deSi encontrado no solo deste estudo é conseqüência do avançado grau de intemperismo em que se encontram os sol os de regiões tropicais (Barbosa Filho et al., 2001) e dos elevados teores de sesquióxidos de Al e Fe (Malavolta, 1980), que são os principais responsáveis pela adsorção de Si em solução(Mengel \& Kirkby, 1987). Outro fator importante que contribui para a diminuição dos teores de Si no solo éa extração do el emento por culturas acumuladoras, o que, via de regra, não é reposto por falta de uso de adubação silicatada (Lima Filho et al., 1999).

No entanto, como já discutido, a aplicação de Si não afetou a produção de matéria seca, oque permite inferir que o teor original do sol o foi suficiente para o desenvolvimento adequado das plantas de arroz do cultivar IAC-202. Contudo, Korndörfer et al . (1999a), trabalhando em casa de vegetação com o cultivar IAC-165, concluíram que o nível de suficiência de Si no solo para obter $90 \%$ da produtividade máxima foi de $9,8 \mathrm{mg} \mathrm{dm}^{-3}$, ou seja, valor acima do teor encontrado no presente estudo sem aplicação de Si. Essa falta de resposta pode estar relacionada com vários fatores, dentre os quais a diferença de cultivares, podendo ser o IAC-165 mais exigente em Si que o IAC-202.

Considerando o teor deSi na planta, houve efeito significativo da interação entre doses de N e Si, estando o desdobramento apresentado no quadro 2. Analisando os efeitos das doses de Si dentro das doses de N, verifica-se efeito significativo deSi para todas as doses deN. Quando a adubação nitrogenada foi baixa, os teores deSi na planta tiveram aumentos mais pronunciados, quando comparados com as doses mais elevadas de N. Analisando o desdobramento dos efeitos de doses de $\mathrm{N}$ dentro das doses de $\mathrm{Si}$, observou-se efeito significativo de $\mathrm{N}$ em todas as doses da adubação silicatada. N otou-se que a aplicação da dose 75 e $150 \mathrm{mg} \mathrm{kg}^{-1}$ de $\mathrm{N}$ propiciou os menores teores de Si na planta em todas as doses da adubação silicatada.

Os teores de Si na planta são classificados como baixos quando menores que $17 \mathrm{~g} \mathrm{~kg}^{-1}$; médios, de 17 a $34 \mathrm{~g} \mathrm{~kg}^{-1}$ e altos acima de $34 \mathrm{~g} \mathrm{~kg}^{-1}$ (K orndörfer et al., 1999c). Observa-se, no quadro 2, que o teor de Si mais alto foi encontrado na menor dose da adubação nitrogenada combinada com a maior dose da adubação silicatada. Assim, à medida que aumentou a dose de $\mathrm{N}$, houve uma diminuição dos teores de Si na planta para todas as doses da adubação silicatada, sendo esses resultados explicados pela competição que existe entre o $\mathrm{H}_{3} \mathrm{SiO}_{4}{ }^{-}$e o $\mathrm{NO}_{3}{ }^{-}$pel os sítios de absorção da planta, conforme foi relatado por Wallace et al. (1976) e Wallace (1989).
Houve efeitosignificativo da interaçãoSi eN para quantidade deSi acumulado nas plantas (Quadro 3). Analisando os efeitos para doses de Si dentro das doses de N, verifica-se efeito significativo de Si para todas as doses de N. Para cada dose da adubação nitrogenada, as maiores quantidades de $\mathrm{Si}$ acumulado foram al cançadas nas doses de $400 \mathrm{e}$ $600 \mathrm{mg} \mathrm{kg}^{-1}$ de $\mathrm{SiO}_{2}$. Analisando o desdobramento dos efeitos de doses de $\mathrm{N}$ dentro das doses de $\mathrm{Si}$, observaram-se efeitos significativos de $\mathrm{N}$ nas doses de 400 e $600 \mathrm{mg} \mathrm{kg}^{-1}$ de $\mathrm{SiO}_{2}$, nos quais o aumento das doses da adubação nitrogenada propiciou diminuição na quantidade de Si acumulado nas plantas.

Como pode ser observado (Quadro 3), a quantidade de Si acumulado na parte aérea das plantas de arroz apresentou a mesma tendência do teor de Si na planta (Quadro 2). Além dos fatores mencionados anteri ormente por Wal lace et al . (1976) e Wallace (1989), outro fator para a queda no teor de Si na planta seria o efeito de diluição provocado peloN. Assim, o incremento da produção de matéria seca da parteaérea em virtude do aumento das doses de N não foi acompanhado de uma absorção de Si na mesma proporção, ocorrendo diminuição do teor desse el emento na planta (Quadro 1).

Quadro 2. Teor de silício na planta, considerando a aplicação de $\mathbf{N}$ e Si (desdobramento das interações significativas da análise de variância)

\begin{tabular}{ccccc}
\hline \multirow{2}{*}{ Dose de N } & \multicolumn{4}{c}{ Dose de silício (mg kg-1 $\left.\mathbf{S i O}_{\mathbf{2}}\right)^{(\mathbf{1})}$} \\
\cline { 2 - 5 } & $\mathbf{0}$ & $\mathbf{2 0 0}$ & $\mathbf{4 0 0}$ & $\mathbf{6 0 0}$ \\
\hline $\mathrm{mg} \mathrm{kg}^{-1}$ & \multicolumn{5}{c}{$\mathrm{g} \mathrm{kg}^{-1}$} \\
5 & $12,2 \mathrm{aC}$ & $24,5 \mathrm{aB}$ & $29,9 \mathrm{aB}$ & $38,1 \mathrm{aA}$ \\
75 & $6,2 \mathrm{~b} \mathrm{C}$ & $11,4 \mathrm{bBC}$ & $14,6 \mathrm{bAB}$ & $20,4 \mathrm{bA}$ \\
150 & $6,8 \mathrm{~b} \mathrm{~B}$ & $10,6 \mathrm{bAB}$ & $13,5 \mathrm{bA}$ & $15,5 \mathrm{cA}$
\end{tabular}

(1) Médias seguidas das mesmas letras minúsculas, na vertical, e maiúsculas, na horizontal, não diferem estatisticamente entre si pelo teste de Tukey a $5 \%$.

Quadro 3. Quantidade de silício acumulado nas plantas, considerando a aplicação de $\mathbf{N}$ e Si (desdobramento das interações significativas da análise de variância)

\begin{tabular}{ccccc}
\hline \multirow{2}{*}{ Dose de $\mathbf{N}$} & \multicolumn{5}{c}{ Dose de silício (mg kg-1 $\left.\mathbf{S i O}_{\mathbf{2}}\right)^{(\mathbf{1})}$} \\
\cline { 2 - 5 } & $\mathbf{0}$ & $\mathbf{2 0 0}$ & $\mathbf{4 0 0}$ & $\mathbf{6 0 0}$ \\
\cline { 2 - 5 } $\mathrm{mg} \mathrm{kg}^{-1}$ & \multicolumn{5}{c}{$\mathrm{g} \mathrm{m}^{2}$} \\
5 & $6,70 \mathrm{aC}$ & $10,61 \mathrm{aB}$ & $18,18 \mathrm{aA}$ & $19,81 \mathrm{aA}$ \\
75 & $4,20 \mathrm{aC}$ & $8,68 \mathrm{aB}$ & $11,11 \mathrm{bAB}$ & $14,54 \mathrm{bA}$ \\
150 & $5,86 \mathrm{aC}$ & $8,72 \mathrm{aBC}$ & $11,91 \mathrm{bAB}$ & $12,97 \mathrm{bA}$ \\
\hline
\end{tabular}

(1) Médias seguidas das mesmas letras minúsculas, na vertical, e maiúsculas, na horizontal, não diferem estatisticamente entre si pelo teste de Tukey $5 \%$. 


\section{CONCLUSÕES}

1. A produção de matéria seca, a produtividade de grãos e os teores de $\mathrm{N}$ na planta de arroz não foram influenciados pelas diferentes doses de $\mathrm{Si}$ aplicadas ao solo.

2. A utilização da adubação silicatada aumentou, de forma significativa, os teores de Si no solo.

3. O aumento da adubação nitrogenada incrementou a produção de matéria seca, a produtividade de grãos e os teores de $\mathrm{N}$ e diminuiu os teores de Si na planta, porém nenhum efeito significativo foi encontrado para os teores de Si no solo.

\section{LITE RATURA CITADA}

AGARIE, S.; HANAOKA, N.; UENO, O.; MIYAZAKI, A.; KUBOTA, F.; AGATA, W. \& KAUFMAN, P.B. Effects of silicon on tolerance to water defict and heat stress in rice plants (Oryza sativa L.), monitored by electrolyte leakage. Plant Prod. Sci., 1:96-103, 1998.

BALASTRA, M.L.F.; PEREZ, C.M.; J ULIANO, B.O.\& VILLREAL, P. Effects of sílica level on some properties of Oriza sativa straw and hult. Can. J . Bot., 67:2356-63, 1989.

BARBOSA FILHO, M.P. Nutrição e adubação do arroz. Piracicaba, Associação Brasileira para Pesquisa da Potassa e do fosfato, 1987. 127p.

BARBOSA FILHO, M.P.; SNYDER, G.H.; ELLIOTT, C.L.; DATNOFF, L.E.; PRABHU, A.S., SILVA, O.F. \& KORNDÖRFER, G.H. Resposta do arroz de sequeiro à aplicação de silício. In: FERTBIO 1998, Caxambu. Anais. Lavras, Universidade Federal de Lavras/Sociedade Brasileira de Ciência do Solo/Sociedade Brasileira de Microbiologia, 1998. p.57

BARBOSA FILHO, M.P.; SNYDER, G.H.; FAGERIA, N.K.; DATNOFF, L.E. \& SILVA, O.F. Silicato de cálcio como fonte de silício para o arroz de sequeiro. R. Bras. Ci. Solo, 25:325-30, 2001

CARVALHO, J.C. Análise de crescimento e produção de grãos da cultura do arroz irrigado por aspersão em função da aplicação de escórias de siderurgia como fonte de silício. Botucatu, Universidade Estadual Paulista 2000. 119p. (Tese de Mestrado)

DEREN, C.W.; DATNOFF, L.E.; SNYDER, G.H. \& MARTIN F.G. Silicon concentration, disease response, and yield components of rice genotypes grown on flooded organic histosols. Crop Sci., 34:733-37, 1994.

EMPRESA BRASILEIRA DE PESQUISA AGROPECUÁRIA EMBRAPA. Centronacional de pesquisa de solos. Sistema brasileiro de classificação de solos. Brasília, 1999. 421p.

FARIA, R.G. Influência do silicato de cálcio na tolerância do arroz de sequeiro ao déficit hídrico do solo. Lavras, Universidade Federal de Lavras 2000. 47p. (Tese de Mestrado)
KORNDÖRFER, G.H.; ARANTES, V,A.; CORRÊA, G.F. \& SNYDER, G.H. Efeito do silicato de cál cio no teor de silício e na produção de grãos de arroz de sequeiro. R. Bras. Ci. Solo, 23:635-41, 1999a

KORNDÖRFER, G.H.; COELHO, N.M.; SNYDER, G.H.\& MIZUTANI, C.T. Avaliação de métodos de extração de silício em solos cultivados com arroz de sequeiro. R. Bras. Ci. Solo, 23:101-106, 1999b.

KORNDÖRFER, G.H.; SNYDER, G.H.; ULLOA, M.; PERDOMO, R.; POWELL, C., DEREN, C. \& DATNOFF, L.E. Soil and plant silicon calibration for rice production. Florida, 1999c. p.14-15 (Manuscript prepared for the Rice Council Meeting, Belle Glade)

LIANG, Y.C.; MA, T.S.; LI, F.J . \& FENG, Y.J . Silicon availability and response of rice and wheat to silicon in calcareous soils. Comm. Soil Sci. Plant Anal., 25:2285-97, 1994.

LIMA FILHO, O.F.; LIMA, M.T.G. \& TSAI, S.M. O silício na agricultura. 1999. p.1-7. (Encarte Técnico - Informe Agronômico, 87)

MALAVOLTA, E. Elementos de nutrição de plantas. Piracicaba, Agronômica Ceres, 1980. 251p.

MALAVOLTA, E. \& FORNASIERI FILHO, D. Nutriçãomineral da cultura do arroz. In: FERREIRA, M.E.; YAMADA, T. \& MALAVOLTA, E. Cultura do arroz de sequeiro fatores afetando a produtividade. Piracicaba, 1983. p.95-143.

MALAVOLTA, E.; VITTI. G.S. \& OLIVEIRA. S.A. Avaliação do estado nutricional das plantas: princípios eaplicações. 2.ed. Piracicaba, POTAF OS, 1997. 319p.

McKEAGUE, J .A. \& CLINE, M.G. Silica in soil solution. II The adsorption of monossilic acid by soil by other substances. Can. J. Soil Sci., 43:83-95, 1963.

MENGEL, K.E.\& KIRKBY, G.A. Further elements of importance. In: PRINCIPLES of plant. 4.ed. WorblaufenBern, International Potash Institute, 1987. p.573-588

RAIJ , B. van. \& CAMARGO, O.A. Sílica solúvel em solos. Bragantia, 32:223-31, 1973.

RAIJ , B. van. \& QUAGGIO, J.A. Métodos de análise de solo para fins de fertilidade. B. Tec. Inst. Agron., 81:1-31, 1983.

RAIJ , B. van.; CANTARELLA, H.; QUAGGIO, J .A. \& FURLANI, A.M.C. Recomendações de adubação e calagem para o estado de São Paulo. 2.ed Campinas, I nstituto Agronômico de Campinas, Fundação IAC, 1996. 285p.

SAVANT, N.K.; DATNOFF, L.E. \& SNYDER, G.H. Depletion of plant-avaibable silicon in soils: a possible cause of declining rice yields. Comm. Soil Sci. Plant Anal., 28:124552, 1997.

TANAKA, A. \& PARK, Y.D. Significance of the absorption and distribution of silica in the growth of rice plant. Soil Sci. Plant Nutr., 12:23-8, 1966.

TISDALE, S.L.; NELSON, W.L.; BESTON, J.D. \& HAULIN, J.L. Soil fertility and fertilizer. New York, Macmillam, 1993. p.634.

WALLACE, A.; ROMNEY, E.A. \& MUELLER, R.T. Nitrogensilicon interaction in plants grown in desert soil with nitrogen deficiency. Agron. J ., 68:529-530, 1976.

WALLACE, A. Relacionships among nitrogen, silicon, and heavy metal uptake by plants. Soil Sci., 147:457-60, 1989. 
WINSLON, M.D. Silicon, disease resistance, and yield of rice genotypes under upland cultural conditions. Crop Sci., 32:1208-1213, 1992.
YOSHIDA, S.; OHNISHI, Y.\& KITAGISHI, K. Chemical forms, mobility and deposition of silicon in rice plant. Soil Sci. Plant Nutr., 8:15-21, 1962. 
M. MAUAD et al.

R. Bras. Ci. Solo, 27:867-873, 2003 